\title{
MÉTHODE DE FROST MODIFIÉE PAR VAN OIJEN
}

par

\section{H. BARKWORTH}

Bactériologiste spécialisé en Laiterie, Collège d'Agriculture du Sud-Est, Wye, Kent.

Les lecteurs de "Le Lait" se souviennent de l'article de ClarenBURG et VAN OIJEN [1], décrivant une adaptation de la méthode de Frost des petites cultures sur lames pour le dénombrement des bactéries dans le lait. Cette méthode est une méthode de laboratoire; mais si on compare les deux méthodes elle est plus à recommander que la numération sur plaques : en effet, le résultat est obtenu en 24 heures, on utilise moins d'appareils et la détermination finale du nombre de bactéries est basée sur l'examen d'une plus grande quantité de lait. La méthode de Van Oijen est surtout une méthode pour le lait de qualité supérieure. Elle donne des résultats jusqu'à 200.000 bactéries par centimètre cube ; mais elle convient spécialement pour les échantillons ne contenant pas plus de 30.000 bactéries par centimètre cube. Toutefois, en diluant le lait, il est possible d'évaluer des contenances plus grandes que 200.000 par centimètre cube.

La méthode de Van Oijen a été étudiée d'une façon critique à Wye; on en a revu la technique et on a comparé avec soin les résultats obtenus par la méthode Van Oijen et la méthode standard de numération sur plaques. Si on doit utiliser une nouvelle méthode, il est nécessaire qu'on sache comment se comportent les résultats obtenus par la nouvelle méthode par rapport à ceux obtenus par les méthodes actuelles; on doit savoir si la nouvelle méthode indique le même (ou à peu près le même) nombre de bactéries. La nouvelle technique doit être susceptible de standardisation, et il est aussi nécessaire de comparer la précision de la nouvelle méthode avec les méthodes utilisées actuellement. Le terme "précision" exige une définition : dans l'étude qui va suivre, ce terme sera utilisé relativement aux essais en double effectués sur le même échantillon. Si les différences entre les essais effectués en double sur le même échantillon sont moindres, la méthode sera considérée comme plus précise. Tous ces points ont été étudiés au laboratoire de Wye.

Pour les lecteurs qui n'auraient pas eu connaissance du précédent article [1], j'indique brièvement comment on opère. Pour chaque échantillon, nous utilisons une lame porte-objets sur laquelle deux rectangles ont été dessinés, chacun de $20 \times 25 \mathrm{~m} / \mathrm{m}$. Avec une pipette convenablement graduée, on verse $1 / 20^{\mathrm{e}}$ de centimètre cube

Traduetion C. Worf. 
de lait dans chaque rectangle, puis on ajoute la même quantité du milieu de culture, on mélange, et on met à l'étuve à $28^{\circ} \mathrm{C}$. Après incubation, les lames sont séchées et colorées, ce qui donne une coloration pourpre aux colonies qui peuvent être ainsi facilement comptées. VAN OIJEN a rendu la numération particulièrement simple en plaçant sur la culture un petit châssis divisé en carrés d'un millimètre carré.

\section{Plan de l'essai.}

Pour pouvoir effectuer une étude complète de la méthode de Van Oijen, des échantillons de lait cru (de diverses fermes) furent examinés cinq fois en même temps par la méthode Van Oijen et par la méthode standard de numération sur plaques [2]. Tous les échantillons de lait avaient 24 heures d'âge quand on les étudiait. Trois points étaient à examiner : la meilleure durée d'incubation ; la comparaison du nombre de bactéries obtenu par cette méthode et par celle de la numération sur plaques; et enfin la précision comparée des deux méthodes.

Dès le début, il était nécessaire de standardiser la technique du test de VAN OIJEN [3]. La méthode est brièvement la suivante :

1. Agiter l'échantillon.

2. Avec une pince, retirer la lame du flacon d'alcool ; la passer à travers la flamme, et quand elle a été stérilisée, la placer sur la table chaude, le côté gravé en dessus.

3. Retirer $1 / 10^{\mathrm{e}}$ de centimètre cube de lait de l'échantillon. Elever et abaisser le niveau du liquide dans la pipette six fois en aspirant et soufflant alternativement, et quand elle est retirée entièrement, enlever le lait à l'extérieur de la pipette en faisant pénétrer la pointe à travers un papier filtre stérile tendu sur un becher ou un bocal de Kilner. (Il est recommandé de percer le papier-filtre avec un canif avant la stérilisation.) Utiliser une pipette pour chaque échantillon.

4. Attendre 60 secondes entre le flambage de la lame et le moment où le lait sera versé sur elle, puis verser $1 / 20^{\mathrm{e}}$ de centimètre cube de lait sur chaque rectangle.

5. Ajouter $1 / 20^{\mathrm{e}}$ de centimètre cube d'agar à chaque rectangle.

6. Passer la spatule trois fois à travers la flamme, attendre 20 secondes, puis mélanger le lait et l'agar, et les étendre en couche régulière sur chaque rectangle. Ne pas passer la spatule à la flamme en passant d'un rectangle à un autre.

7. Poser la lame sur une surface préalablement nivelée et couvrir pour protéger de la poussière. Après 10 minutes de repos, placer à l'étuve.

8. Stériliser la spatule. 
Une analyse statistique des résultats comportant une comparaison du nombre des colonies sur chaque rectangle de $20 \times 25 \mathrm{~m} / \mathrm{m}$ indique que la technique opératoire ci-dessus décrite est bonne.

Ayant fixé cette standardisation de la technique, nous avons ensuite comparé les résultats obtenus en faisant varier les durées d'incubation. Des échantillons de lait furent essayés cinq fois, et les résultats furent examinés au point de vue statistique : ces résultats indiquèrent que 24 heures à $28^{\circ} \mathrm{C}$ constituent la durée d'incubation standard pour le test de Van Oijen.

Dans le test de Van Oijen, l'augmentation du nombre de colonies entre 9 heures et 24 heures, et aussi entre 17 heures et 24 heures est très nette au point de vue statistique. Il est exact qu'aussi bien dans la numération sur plaques que dans le test de Van Oijen, des augmentations ultérieures du nombre des colonies peuvent être obtenues par une incubation prolongée, mais pour un travail normal ce serait impraticable. En outre, l'augmentation obtenue par une incubation prolongée est très inférieure à celle obtenue entre 17 heures et 24 heures ou entre 9 heures et 24 heures. Il est certain que l'incubation des lames Van Oijen à $28^{\circ} \mathrm{C}$. pendant moins de 24 heures serait susceptible de donner des résultats pouvant induire en erreur.

Les résultats des tests pour des durées d'incubation variables, exprimés en pourcentage, sont donnés ci-dessous. On verra qu'en 9 heures nous n'avons obtenu que $78 \%$ des colonies obtenues en 24 heures d'incubation, et que la même différence se présente entre 17 heures et 24 heures.

\section{Lait du matin.}

Test de Van Oijen

9 heures à $28^{\circ} \mathrm{C}$.

24 heures à $28^{\circ} \mathrm{C}$.

\section{$\%$}

78

100
Numération en plaques

72 heures à $37^{\circ} \mathrm{C}$.

72 heures plus 72 heures à $22^{\circ} \mathrm{C}$.
$\%$

100

112

\section{Lait de I'après-midi.}

17 heures à $28^{\circ} \mathrm{C}$.

24 heures à $28^{\circ} \mathrm{C}$.

24 heures à $28^{\circ} \mathrm{C}$.

72 heures à $37^{\circ} \mathrm{C}$.

plus 72 heures à $22^{\circ} \mathrm{C}$.

Ces chiffres nous amenèrent à comparer les résultats de l'incubation des lames de Van Oijen pendant 24 heures à $28^{\circ} \mathrm{C}$. avec ceux de l'incubation de culture sur plaques pendant 72 heures à $37^{\circ} \mathrm{C}$., et des essais répétés cinq fois furent exécutés sur cette base.

Les résultats doivent être classés en deux groupes :

a) Echantillons avee une numération de 3.000 à 30.000 par centimètre cube.

b) Echantillons avec une numération de 30.000 à 300.000 par centimètre cube. 
Il était nécessaire de procéder à ce classement, parce qu'aussi bien dans la numération sur plaques que dans le test de Van Oijen, le résultat final dans chaque groupe est basé sur une quantité différente de lait. Dans le groupe $a$ ) la numération est basée sur le nombre de colonies sur une plaque d'une dilution au $1 / 100^{\mathrm{e}}$, tandis que dans le groupe $b$ ), elle est basée sur une plaque d'une dilution au $1 / 1000^{\mathrm{e}}$. En d'autres termes, dans la numération sur plaques, nous examinons dans le groupe a) la croissance de $1 / 100^{\mathrm{e}}$ de centimètre cube de lait et dans le groupe $b$ ) de $1 / 1000^{\mathrm{e}}$ de centimètre cube de lait. Une différence parallèle a lieu dans le test de Van Oijen, mais pour comprendre la différence dans ce cas, nous devons tenir compte de la méthode de numération des colonies dans le test de Van Oijen qui est la suivante : un châssis gravé en carrés de $1 \mathrm{~m} / \mathrm{m}$ carré est placé sur chaque rectagle. Chaque rectangle a $20 \times 25 \mathrm{~m} / \mathrm{m}$ et contient par conséquent 20 rangées de 25 carrés, et comme chaque rectangle contient $1 / 20^{\mathrm{e}}$ de centimètre cube de lait, il s'ensuit que chaque millimètre carré représente $1 / 10.000^{\mathrm{e}}$ de centimètre cube de lait.

Pour les laits à faible numération, nous comptons toutes les colonies dans une rangée sur deux, et comme il y a deux rectangles, si nous inspectons une rangée sur deux dans les deux rectangles, nous avons finalement inspecté la moitié du lait, soit $2 \times 1 / 40^{\mathrm{e}}$ centimètre cube de lait $=1 / 20^{\mathrm{e}}$ de centimètre cube de lait.

Pour les numérations élevées, groupe $a$ ), nous comptons les colonies dans 25 carrés choisis sur chaque rectangle, ce qui fait que nous examinons $1 / 200^{e}$ de centimètre cube de lait. On notera que, dans chaque groupe, la quantité de lait examinée en vue de la numération est plus grande dans le test de Van Oijen que dans le groupe correspondant de la numération en plaques.

L'analyse statistique des résultats montre que, dans les deux groupes, le test de Van Oijen est très nettement le plus précis. On peut soutenir que le test de Van Oijen doit être plus précis parce que le résultat est basé sur l'examen d'une plus grande quantité de lait. Ce point n'a pas échappé à l'examen, et des calculs ultérieurs ont été effectués qui indiquent que la précision accrue du test de Van Oijen est supérieure à celle qui peut être attribuée à la plus grande quantité de lait examiné. Si on le compare à la numération sur plaques, le test de Van Oijen est plus précis, non pas seulement comparativement, mais en valeur absolue.

Il faut noter un autre point : les résultats obtenus par le test de Van Oijen tendent à être légèrement, mais nettement, inférieurs à ceux obtenus par la numération sur plaques. Quoique nette au point de vue statistique, la différence est trop petite pour être d'importance pratique. 
Les chiffres moyens sont les suivants :

Moyenne d'échantillons à faible numération,

$\begin{array}{cc}\text { Test de } & \text { Numération } \\ \text { Van Oijen } & \text { en plaques }\end{array}$

$$
4,000 \quad 4,500
$$

Moyenne d'échantillons à numération élevée, groupe $b) \ldots \ldots \ldots \ldots \ldots \ldots \ldots \ldots \ldots \ldots \ldots$

$$
50,100 \quad 74,100
$$

Dans sa forme actuelle, le test de Van Oijen ne donne que le nombre de colonies par centimètre cube de lait ; mais il ne serait pas difficile de combiner ce test avec le test des organismes coliformes, c'est-à-dire l'ensemencement sur bouillon lactose-bile-sel-peptone. Nous ne voulons pas discuter ici des mérites et des inconvénients du test des organismes coliformes quand on l'applique au lait : qu'il nous suffise de dire que nombreux sont les expérimentateurs qui considèrent que les deux tests, numération totale et test des coliformes, devraient être effectués ensemble. Le soussigné a imaginé des méthodes simples, susceptibles d'être employées dans la pratique courante et qui combinent le test de Van Oijen avec le test des coliformes. Il reste à contrôler la précision de la nouvelle technique du test des coliformes comparée aux méthodes officielles actuelles.

VAN OIJEN a simplifié la numération par l'introduction du châssis quadrillé ; mais le test pourrait facilement être encore plus simplifié et rendu plus automatique. On pourrait utiliser un châssis suffisamment grand pour couvrir les deux rectangles, et lorsque le test est régulièrement utilisé, on pourrait avantageusement avoir des supports de dimensions convenables qui soutiendraient les lames pendant l'incubation, la coloration, le lavage et le séchage final.

\section{Résumé}

La méthode de Van Oijen modifiée a été comparée avec la méthode standard de numération sur plaques. Les lames porte-objets de Van Oijen doivent être mises à l'étuve pendant 24 heures à $28^{\circ} \mathrm{C}$. La méthode de Van Oijen est plus précise que la numération sur plaques.

\section{BIBLIOGRAPHIE}

[1] A. Clarenburg et C. F. Van Oijen. Recherches systématiques sur la méthode de Frost des petites cultures sur lames pour le dénombrement des bactéries vivantes dans le lait. Le Lait, t. VII, avril $1927, \mathrm{n}^{\circ} 64$, p. 321-336.

[2] Ministry of Agriculture : Guide to the Conduct of Clean Milk competitions, bull. $n^{\circ} 46,5$ th, Ed., février 1934.

[3] H. Barkworth. The Technique of Van Oijen (modified) test. Journal S. E. Agricultural College, no 38, juillet 1936, p. 143-149. 